\title{
Comparison of Conservative versus Surgical Treatment Protocols in Treating Idiopathic Granulomatous Mastitis: A Meta-Analysis
}

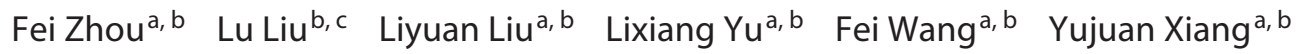 \\ Chao Zheng $^{a, b}$ Shuya Huang ${ }^{a, b}$ Han Cai ${ }^{a, b}$ Zhigang Yu ${ }^{a, b}$ \\ ${ }^{a}$ Department of Breast Surgery, The Second Hospital of Shandong University, Jinan, China; ${ }^{\mathrm{b}}$ Institute of \\ Translational Medicine of Breast Disease Prevention and Treatment, Shandong University, Jinan, China; \\ 'Department of Breast Surgery, Qingdao Municipal Hospital Group, Qingdao, China
}

\section{Keywords}

Idiopathic granulomatous mastitis - Corticosteroids .

Surgery $\cdot$ Treatment $\cdot$ Meta-analysis

\begin{abstract}
Background: Idiopathic granulomatous mastitis (IGM) is a rare, benign breast disease without any definitive therapeutic strategy. It is controversial whether to use conservative or surgical treatment of IGM and high-level evidence-based medicine data are lacking. The purpose of this study was to systemically evaluate the clinical effectiveness of the conservative versus surgical treatment for IGM. Methods: In this meta-analysis, we searched PubMed, EMbase, ScienceDirect, and Web of Science for comparative studies about the conservative versus surgical treatment of IGM. Two researchers independently identified reports and extracted data. We used Stata 11 for data analysis. A meta-analysis was performed to investigate the differences in the recurrence rate of conservative and surgical treatment using a random effects model. Results: A total of 10 studies involving 1,101 patients were included. The results demonstrated that there was no significant difference in the recurrence rate among patients who were treated by nonsurgical therapy and surgical treatment. No publication bias was detected. Conclusion: This meta-analysis demonstrates that both the conser-
\end{abstract}

vative and surgical treatment approaches have appropriate efficacy in IGM treatment and relapse. Further randomized controlled trials with longer follow-up periods are required to confirm the advantages of each approach.

(c) 2019 S. Karger AG, Basel

\section{Introduction}

Idiopathic granulomatous mastitis (IGM) is a rare, benign, chronic inflammatory disease of the breast and was first described by Kessler and Wolloch [1] in 1972. The etiology of IGM remains unclear; autoimmune and microbial infection, hormonal effect, local trauma, and alpha- 1 antitrypsin deficiency are proposed as some critical risk factors contributing to IGM [2]. It generally emerges with the clinical symptoms of breast mass, abscess, inflammation, and mammary duct fistula [3]. The ultrasound examination and mammographic findings are considered nonspecific and similar to those of breast cancer [4]. Pathological findings are the goal standard for IGM diagnosis with a chronic non-necrotizing granulomatous inflammation in lobules of the breast tissue [5].

There is no consensus on the optimal therapeutic strategy for IGM. Surgical removal of the diseased section is the most commonly recommended approach but shows 
Table 1. Characteristics of the included studies

\begin{tabular}{|c|c|c|c|c|c|}
\hline First author [ref.] & $\begin{array}{l}\text { Year of } \\
\text { publication }\end{array}$ & Country & $\begin{array}{l}\text { Conservative } \\
\text { recurrence/total }\end{array}$ & $\begin{array}{l}\text { Surgical } \\
\text { recurrence/total }\end{array}$ & $\begin{array}{l}\text { Median follow-up } \\
\text { time, months }\end{array}$ \\
\hline Aldaqal [12] & 2004 & Saudi Arabia & $0 / 6$ & $4 / 19$ & 25 \\
\hline Wilson [13] & 2007 & United States & $21 / 38$ & $16 / 78$ & 13 \\
\hline Kuba [14] & 2009 & Japan & $8 / 31$ & $38 / 169$ & 12 \\
\hline Kayahan [15] & 2012 & Turkey & $1 / 12$ & $4 / 18$ & 42 \\
\hline Oran [16] & 2013 & Turkey & $5 / 25$ & $3 / 21$ & 35 \\
\hline Omranipour [17] & 2013 & Iran & $7 / 22$ & $0 / 21$ & 16 \\
\hline Yabanoğlu [18] & 2015 & Turkey & $9 / 44$ & $0 / 33$ & 17 \\
\hline Atak [19] & 2015 & Turkey & $2 / 17$ & $13 / 23$ & 25 \\
\hline Aghajanzadeh [20] & 2015 & Iran & $11 / 195$ & $0 / 11$ & 18 \\
\hline Uysal [21] & 2017 & Turkey & $37 / 258$ & $12 / 60$ & 16 \\
\hline
\end{tabular}

more cosmetic problems [6]. Conservative alternatives include close observation, antibiotics, or immunosuppressive therapy with corticosteroids. However, varying rates of recurrence are reported to be as high as 77\% [7]. A few studies demonstrate the advantages and disadvantages of both treatment protocols in a large patient population, but there are few studies to date comparing the efficiency of these two treatments that use the meta-analysis method. This meta-analysis makes a comparison between two different therapeutic methods comprising surgical treatment protocols and conservative approaches, which include observation and a special pharmaceutical regimen without surgical approach.

\section{Materials and Methods}

\section{Search Strategy}

A comprehensive search was conducted for relevant articles published between January 1981 and April 2018 from the following database: (1) PubMed; (2) EMbase; (3) ScienceDirect; and (4) Web of Science. Search terms included "granulomatous mastitis," "granulomatous lobular mastitis," "non-puerperal mastitis," and "breast abscesses." Moreover, the reference lists of retrieved articles were scrutinized to identify further relevant studies. In some studies, the corresponding authors were contacted for more information beyond what was in their published articles.

\section{Eligibility Criteria}

All identified studies were independently reviewed by two investigators to determine whether an individual study was eligible for inclusion in this meta-analysis. The inclusion criteria were as follows: (1) patients with the histologic diagnosis of IGM; (2) comparative data between conservative and surgical treatment protocols were available; (3) studies with a number of cases $>20$; (4) the median follow-up was longer than 6 months. If there was disagreement between the two investigators about the eligibility of an article, it was resolved by consensus with a third reviewer.

\section{Data Extraction}

The data of included articles were extracted by two investigators independently. The conservative management included oral steroids, MTX, antibiotics, and observation; the surgical treatment contained drainage, lumpectomy, wide local excision, and mastectomy. Recurrence was defined as the reappearance of inflammatory skin changes, lump, ulcer, and fistula after complete remission. Any discrepancies were resolved by discussion until a consensus was reached. The following data were collected from each study: the first author's name, year of publication, countries where the studies were performed, sample size (number of patients in the conservative-treatment and surgical groups), recurrence rate for each treatment protocol, and duration of follow-up.

All of our included studies were observational comparative studies, and the methodological quality was assessed by the Newcastle-Ottawa Scale (NOS) [8]. A score between 0 and 9 was assigned to each study.

\section{Statistical Analysis}

The Stata 11.0 was used to perform statistical analysis. Dichotomous variables were presented as odds ratios (OR) and 95\% confidence intervals (CI). We evaluated statistical heterogeneity using the $\chi^{2}$ test to assess heterogeneity between trials with a $p$ value $<0.10$. The $I^{2}$ of Higgins and Thompson was used to assess heterogeneity among studies [9]. $I^{2}$ describes the proportion of the total variation attributable to between-study heterogeneity as opposed to random error or chance. In the presence of substantial heterogeneity $\left(I^{2}>50 \%\right)[10]$, the DerSimonian and Laird random effect model (REM) was adopted as the pooling method; otherwise, the fixed effect model (FEM) was used as the pooling method. We intended to conduct a prespecified subgroup analysis of outcomes for different locations. Publication bias was estimated using Begg's rank correlation test [11].

\section{Results}

\section{Study Characteristics}

The results of the literature search identified 1,514 papers based on the search words. Out of these, based on the criteria described earlier, 10 publications involving 1,101 patients were eligible for inclusion in this meta-analysis [12-21]. All involved articles were retrospective studies, and the median follow-up ranged from 12 to 42 months. Of the 10 articles, 5 studies were conducted in Turkey [15, $16,18,19,21], 2$ in Iran $[17,20]$, and the other 3 in Saudi Arabia [12], Japan [14], and the United States [13]. The 
Fig. 1. Study selection.

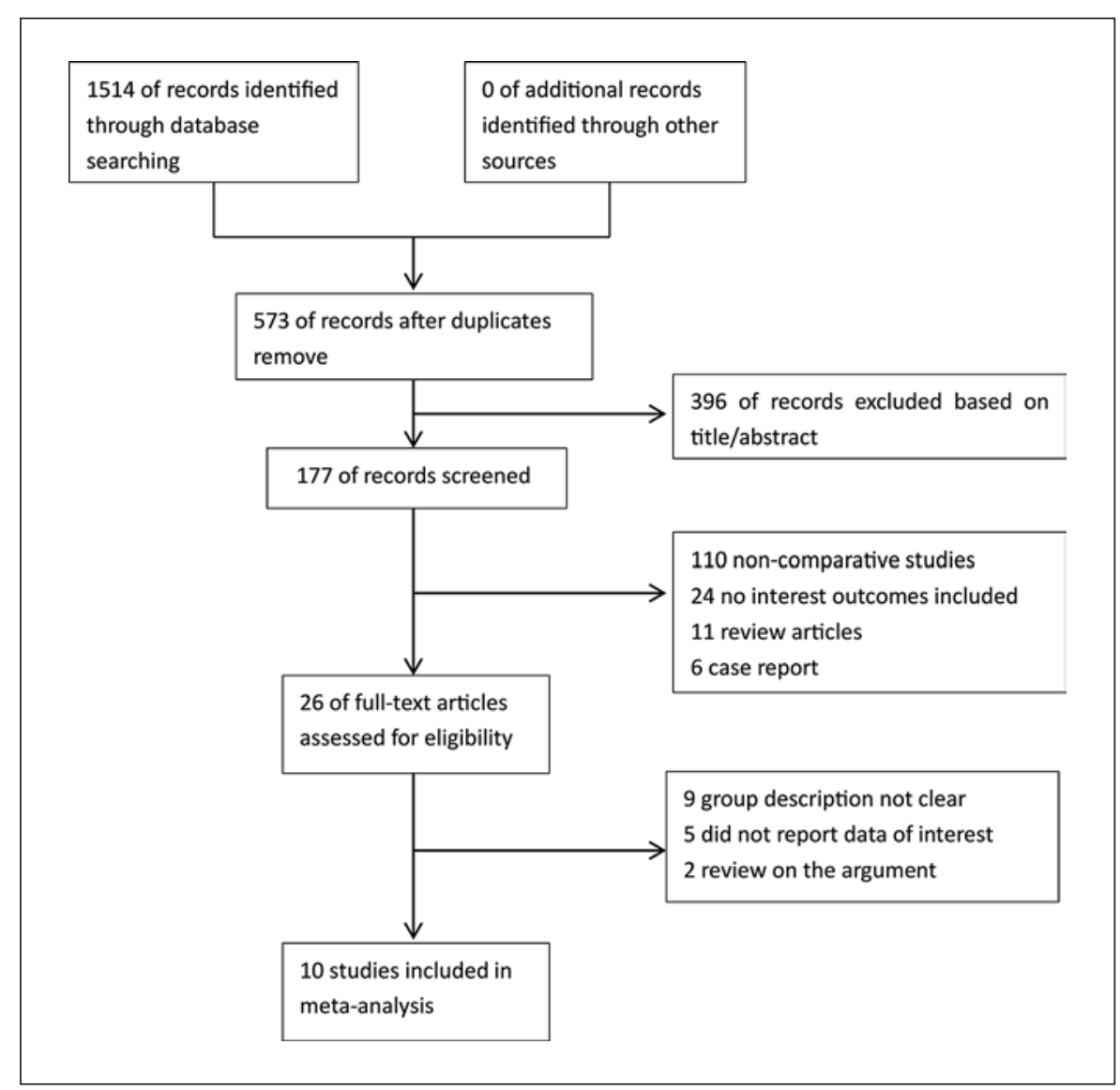

details of the study selection and basic characteristics of the included studies are shown in Table 1 and Figure 1. No randomized trial was included, so the quality of studies was assessed through the NOS. Overall, there was an average medium quality of 6 out of 9 stars in all studies (range 3-7).

\section{Recurrence Rate}

All included studies provided recurrence rate data, and the OR and 95\% CI for each study and the summary OR are shown in Figure 2. The overall estimated OR was 1.25 (95\% CI: 0.51-3.03, $p=0.629$ ). Using a random effect model, heterogeneity testing revealed $I^{2}=70.3 \%$ and $p$ for heterogeneity $<0.001$. There was no significant difference in the recurrence rate between the conservative and surgical treatment groups. We introduced two subgroups according to the location. Eight articles [12, 15-21] were included into the "developing-country subgroup" because all of them were conducted in developing countries, while the rest of the articles $[13,14]$ were included into the "developed-country subgroup." Heterogeneity still existed within both subgroups $\left(p=0.013, I^{2}=60.5 \%\right.$; $p=$ $0.026, I^{2}=79.8 \%$ ), hence the random effects model was used. Subgroup analysis still revealed no significant difference (95\% CI: 0.32-2.87, $p=0.929$; 95\% CI: 0.62-9.36, $p=0.204)$.

\section{Publication Bias}

No significant publication bias was observed after sensitivity analysis (Fig. 3).

\section{Discussion}

IGM is defined as a rare, chronic, inflammatory, and benign disease mimicking breast carcinoma. The etiology of IGM is not precisely clarified; however, autoimmune and oral contraceptive usage, infectious microorganisms, trauma, hyperprolactinemia, and alpha- 1 antitrypsin deficiency are some critical risk factors contributing to IGM cases [2].

IGM predominantly affects females of childbearing age with a history of pregnancy and lactation in the previous 5 years. The clinical presentation is usually a unilateral, distinct and painful mass. Nonetheless, cutaneous lesions, nipple retraction, lymphadenopathy, abscess, fistula, and sinus formation are other possible symptoms. Ultrasonographic, mammographic, and even magnetic resonance imaging are not pathognomonic.

The pathological-finding approach is recognized as the gold standard for IGM diagnosis, characterized by non-caseating granulomas concentrated in lobules. Microscopically, the granulomas contain Langhans giant 


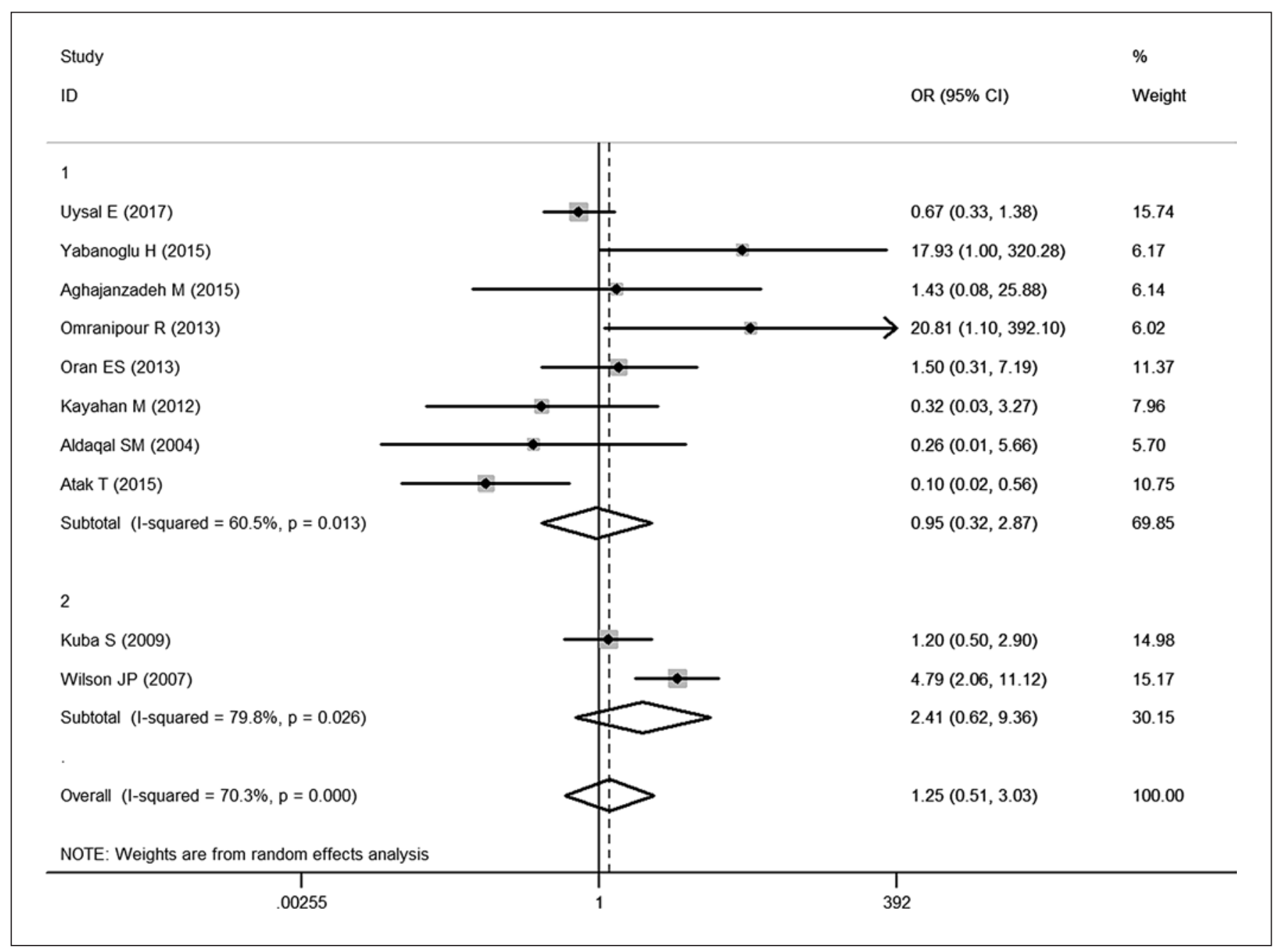

Fig. 2. Forest plot of OR analysis of the recurrence rate of conservative and surgical approaches in treating IGM. Open diamonds denote the pooled OR. Black squares indicate the OR in each study, with the square sizes inversely proportional to the standard error of the OR. Horizontal lines represent the $95 \%$ CI.

Fig. 3. Funnel plot for publication bias.

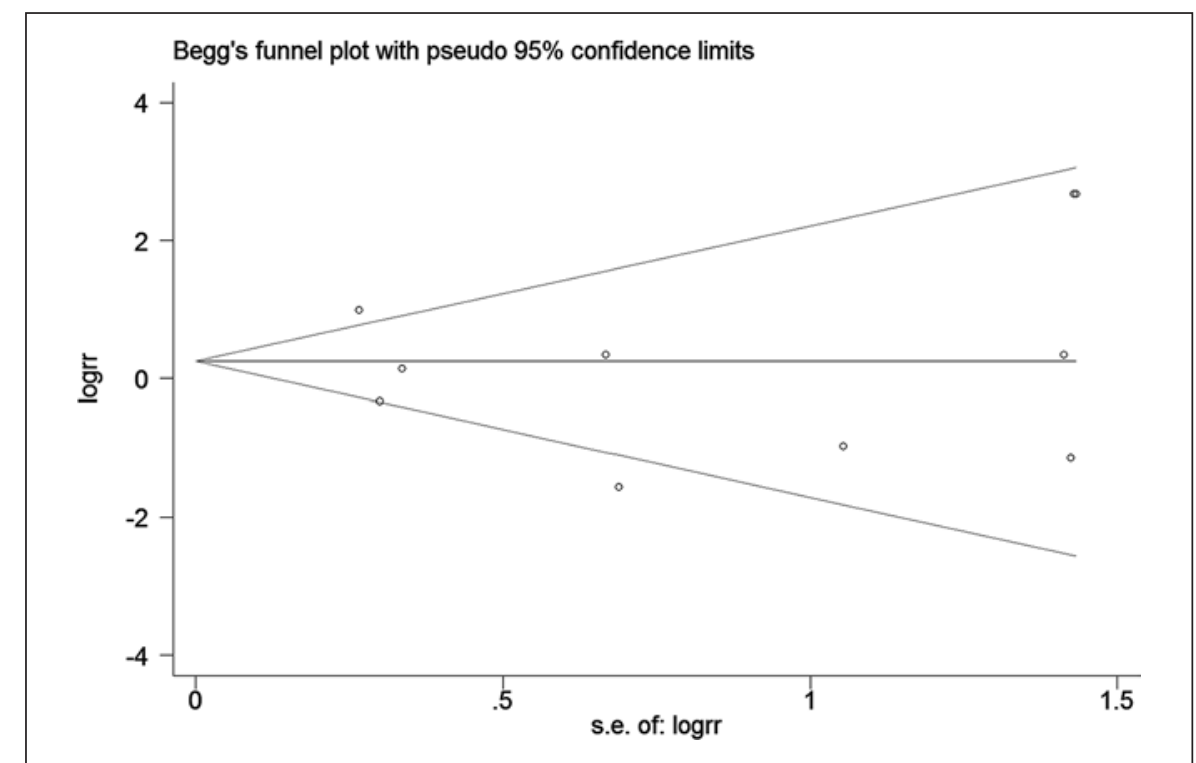


cells, epithelioid histiocytes, and are associated with lymphocytes and plasma cells. Neutrophilic microabscesses may also be seen. Cystic vacuoles are often present within the granulomas and are lined by neutrophils. Gram-positive coryneform bacilli may be present within the cysts. Therefore, pathological analysis is strictly recommended to exclude malignancy.

There is no definitive therapeutic strategy for IGM; treatment methods include mastectomy, excision, drainage, antibiotic therapy, corticosteroid treatment, and observation. Surgical treatment was the mainstream of treatment before 1980, and is still preferred by many experts nowadays [22]. However, the risk of recurrence has limited its application, and multiple surgeries may be required to achieve complete remission.

Furthermore, negative-margin surgery affects patients with inevitable side-effects such as cosmetic issues. As suggested by Neel et al. [23], the first-line surgical therapy did not reduce the incidence of relapse in a long-term follow-up; $80 \%$ of the GLM patients relapsed after surgical treatment, and unsightly scars tended to be more frequent among women who had undergone surgery. Mastectomy is also performed with breast reconstruction in some rare cases $[13,24]$ and imposes huge psychological trauma and financial costs.

In 1980, DeHertogh et al. [25] were the first to recommended that corticosteroid is an appropriate option for the treatment of IGM. Furthermore, Sakurai et al. [26] suggested that corticosteroids could be efficient in $87 \%$ of the patients without any recurrence. In recent years, low doses of corticosteroid were considered effective for IGM patients [27-29]. However, there is some research showing different results. Azlina et al. [30] found that about half of the cases relapsed after discontinuation or decreasing the steroid dose. The proceeded results were validated by Neel et al. [23], whose research implied that corticosteroids were often effective (85\%), but relapse was frequent when the dose was tapered (46\%). For patients with steroidal side effects or who have a resistance to steroids, hydroxychloroquine, methotrexate, azathioprine, and/or colchicines can be used. Antibiotic therapy is not widely analyzed in IGM, but in the case of abscess or sinus formation, empiric antibiotic treatment is recommended due to the high probability of Corynebacterium infection [31].

There is little scientific evidence that can guide the therapeutic management of IGM, which remains controversial. Indeed, some authors recommend conservative treatment, whereas others favor surgery [22, 32, 33]. Therefore, we perform this meta-analysis to make a comparison between two different therapeutic methods comprising the surgical approach, and a special pharmaceutical regimen, which includes corticosteroids and antibiotics, and immunosuppressive therapy without surgical approach. In conclusion, we demonstrate that there is no significant difference in the recurrence of IGM between the surgical and conservative approaches, whereas the implementation of numerous surgeries is associated with a higher probability of wound infection, sinus formation, and other cosmetic problems. Hence, the pharmaceutical approach (without surgery) is strongly recommended; nonetheless, surgery could be suggested in case of recurrent patients or lack of pharmaceutical efficacy.

\section{Acknowledgements}

We thank Xiaohui Yan for reviewing the report contextually and grammatically, and the team from Shandong University library for their support in the database search and acquisition of the majority of included papers.

\section{Statement of Ethics}

The authors have no ethical conflicts to disclose.

\section{Disclosure Statement}

The authors have no conflicts of interest to declare.

\section{Funding Sources}

This work was supported by the National Health and Family Planning Commission's Public-Benefit Project (Grant No. 201502027); and Jinan City Science and Technology Innovation Program (Grant No. 201602146).

\section{Author Contributions}

Fei Zhou: data search, inclusion and exclusion of studies, metaanalysis, main writer. Lu Liu: data search, inclusion and exclusion of studies, main writer. Liyuan Liu: data search, methodological quality assessment, meta-analysis. Lixiang Yu: data search, metaanalysis, article revision. Fei Wang: data extraction, methodological quality assessment, meta-analysis. Yujuan Xiang: data search, data extraction. Chao Zheng: data search, data extraction. Shuya Huang: data extraction, article revision. Han Cai: data search, article revision. Zhigang Yu: study design, supervised data collection and interpretation.

\footnotetext{
References $\quad 1$ Kessler E, Wolloch Y. Granulomatous mastitis: a lesion clinically simulating carcinoma. Am J Clin Pathol. 1972 Dec;58(6):642-6.

2 Altintoprak F, Kivilcim T, Ozkan OV. Aetiology of idiopathic granulomatous mastitis. World J Clin Cases. 2014 Dec;2(12):852-8.

3 Benson JR, Dumitru D. Idiopathic granulomatous mastitis: presentation, investigation and management. Future Oncol. 2016 Jun; 12(11):1381-94.
} 
4 Oztekin PS, Durhan G, Nercis Kosar P, Erel S, Hucumenoglu $\mathrm{S}$. Imaging Findings in $\mathrm{Pa}$ tients with Granulomatous Mastitis. Iran J Radiol. 2016 May;13(3):e33900.

5 Johnstone KJ, Robson J, Cherian SG, Wan Sai Cheong J, Kerr K, Bligh JF. Cystic neutrophilic granulomatous mastitis associated with Corynebacterium including Corynebacterium kroppenstedtii. Pathology. 2017 Jun; 49(4):405-12.

6 Giacalone PL, Rathat G, Fournet S, Rouleau C. Surgical treatment of recurring subareolar abscess using oncoplastic techniques. J Visc Surg. 2010 Dec;147(6):e389-94.

7 Sheybani F, Sarvghad M, Naderi HR, Gharib M. Treatment for and clinical characteristics of granulomatous mastitis. Obstet Gynecol. 2015 Apr;125(4):801-7.

8 Higgins Jp T, Green S. Cochrane Handbook for Systematic Reviews of Interventions. Version 5.1.0. Wiley-Blackwell; 2011. pp. 102-8.

9 Higgins JP, Thompson SG. Quantifying heterogeneity in a meta-analysis. Stat Med. 2002 Jun;21(11):1539-58.

10 Higgins JP, Thompson SG, Deeks JJ, Altman DG. Measuring inconsistency in meta-analyses. BMJ. 2003 Sep;327(7414):557-60.

11 Begg CB, Mazumdar M. Operating characteristics of a rank correlation test for publication bias. Biometrics. 1994 Dec;50(4):1088-101.

12 Aldaqal SM. Idiopathic granulomatous mastitis. Clinical presentation, radiological features and treatment. Saudi Med J. 2004 Dec; 25(12):1884-7.

13 Wilson JP, Massoll N, Marshall J, Foss RM, Copeland EM, Grobmyer SR. Idiopathic granulomatous mastitis: in search of a therapeutic paradigm. Am Surg. 2007 Aug;73(8): 798-802.

14 Kuba S, Yamaguchi J, Ohtani H, Shimokawa I, Maeda S, Kanematsu T. Vacuum-assisted biopsy and steroid therapy for granulomatous lobular mastitis: report of three cases. Surg Today. 2009;39(8):695-9.
15 Kayahan M, Kadioglu H, Muslumanoglu M. Management of Patients with Granulomatous Mastitis: analysis of 31 Cases. Breast Care (Basel). 2012 Jun;7(3):226-30.

16 Oran EŞ, Gürdal SÖ, Yankol Y, Öznur M, Calay Z, Tunacı M, et al. Management of idiopathic granulomatous mastitis diagnosed by core biopsy: a retrospective multicenter study. Breast J. 2013 Jul-Aug;19(4):411-8.

17 Omranipour R, Mohammadi SF, Samimi P. Idiopathic granulomatous lobular mastitis report of 43 cases from iran; introducing a preliminary clinical practice guideline. Breast Care (Basel). 2013 Dec;8(6):439-43.

18 Yabanoğlu H, Colakoğlu T, Belli S, Aytac HO, Bolat FA, Pourbagher A, et al. A Comparative Study of Conservative versus Surgical Treatment Protocols for 77 Patients with Idiopathic Granulomatous Mastitis. Breast J. 2015 JulAug;21(4):363-9.

19 Atak T, Sagiroglu J, Eren T, Ali Özemir I, Alimoglu O. Strategies to treat idiopathic granulomatous mastitis: retrospective analysis of 40 patients. Breast Dis. 2015;35(1):19-24.

20 Aghajanzadeh M, Hassanzadeh R, Alizadeh Sefat S, Alavi A, Hemmati H, Esmaeili Delshad MS, et al. Granulomatous mastitis: Presentations, diagnosis, treatment and outcome in 206 patients from the north of Iran. Breast. 2015 Aug;24(4):456-60.

21 Uysal E, Soran A, Sezgin E; Granulomatous Mastitis Study Group. Factors related to recurrence of idiopathic granulomatous mastitis: what do we learn from a multicentre study? ANZ J Surg. 2018 Jun;88(6):635-9.

22 Hovanessian Larsen LJ, Peyvandi B, Klipfel N, Grant E, Iyengar G. Granulomatous lobular mastitis: imaging, diagnosis, and treatment. AJR Am J Roentgenol. 2009 Aug;193(2):57481.

23 Néel A, Hello M, Cottereau A, Graveleau J, De Faucal P, Costedoat-Chalumeau N, et al. Long-term outcome in idiopathic granulomatous mastitis: a western multicentre study. QJM. 2013 May;106(5):433-41.
24 Yau FM, Macadam SA, Kuusk U, Nimmo M, Van Laeken N. The surgical management of granulomatous mastitis. Ann Plast Surg. 2010 Jan;64(1):9-16.

25 DeHertogh DA, Rossof AH, Harris AA, Economou SG. Prednisone management of granulomatous mastitis. N Engl J Med. 1980 Oct; 303(14):799-800.

26 Sakurai K, Fujisaki S, Enomoto K, Amano S, Sugitani M. Evaluation of follow-up strategies for corticosteroid therapy of idiopathic granulomatous mastitis. Surg Today. 2011 Mar; 41(3):333-7.

27 Asoglu O, Ozmen V, Karanlik H, Tunaci M, Cabioglu N, Igci A, et al. Feasibility of surgical management in patients with granulomatous mastitis. Breast J. 2005 Mar-Apr;11(2):10814.

28 Maffini F, Baldini F, Bassi F, Luini A, Viale G. Systemic therapy as a first choice treatment for idiopathic granulomatous mastitis. J Cutan Pathol. 2009 Jun;36(6):689-91.

29 Erozgen F, Ersoy YE, Akaydin M, Memmi N, Celik AS, Celebi F, et al. Corticosteroid treatment and timing of surgery in idiopathic granulomatous mastitis confusing with breast carcinoma. Breast Cancer Res Treat. 2010 Sep;123(2):447-52.

30 Azlina AF, Ariza Z, Arni T, Hisham AN. Chronic granulomatous mastitis: diagnostic and therapeutic considerations. World J Surg. 2003 May;27(5):515-8.

31 Akbulut S, Arikanoglu Z, Senol A, Sogutcu N, Basbug M, Yeniaras E, et al. Is methotrexate an acceptable treatment in the management of idiopathic granulomatous mastitis? Arch Gynecol Obstet. 2011 Nov;284(5):1189-95.

32 Bani-Hani KE, Yaghan RJ, Matalka II, Shatnawi NJ. Idiopathic granulomatous mastitis: time to avoid unnecessary mastectomies. Breast J. 2004 Jul-Aug;10(4):318-22.

33 Lai EC, Chan WC, Ma TK, Tang AP, Poon CS, Leong HT. The role of conservative treatment in idiopathic granulomatous mastitis. Breast J. 2005 Nov-Dec;11(6):454-6. 